J3eA, Journal sur l'enseignement des sciences et technologies de l'information et des systèmes, Volume 4, Hors-Série 1, 7 (2005)

DOI : http://dx.doi.org/10.1051/bib-j3ea:2005607

(C) EDP Sciences, 2005

Commande d'un système de génération électrique pour réseau de bord d'avion Introduction de charges non linéaires et mise en place d'un filtre LC

C. Khatounian ${ }^{1}$, F. Khatounian ${ }^{1}$, E. Monmasson ${ }^{1}$, F. Berthereau ${ }^{2}$ et J.P. Louis ${ }^{3}$

${ }^{1}$ SATIE, Université de Cergy, rue d'Eragny, F-95301 Cergy-Pontoise, France

2 THALES AES Society, 41 boulevard de la République, F-78401 Chatou, France

${ }^{3}$ SATIE, ENS de Cachan, 61 avenue du Président Wilson, F-94230 Cachan, France

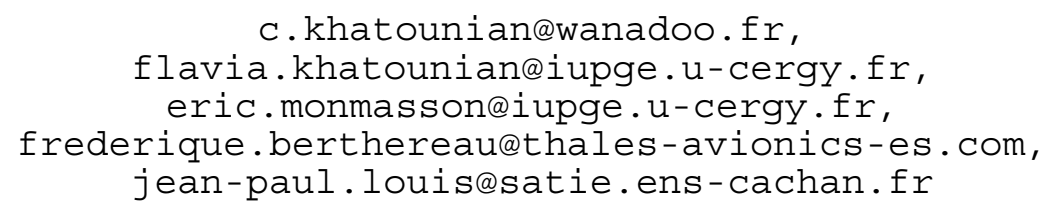




\title{
Commande d'un système de génération électrique pour réseau de bord d'avion - Introduction de charges non linéaires et mise en place d'un filtre LC
}

\author{
C. Khatounian ${ }^{(1)}$, F. Khatounian ${ }^{(2)}$, E. Monmasson ${ }^{(3)}$, F. Berthereau ${ }^{(4)}$, J.P. Louis ${ }^{(5)}$ \\ (1) (2) (3) SATIE - Université de Cergy, rue d'Eragny, 95301 Cergy-Pontoise, France, \\ emails : c.khatounian@wanadoo.fr, flavia.khatounian@iupge.u-cergy.fr, eric.monmasson@iupge.u-cergy.fr \\ (4) THALES AES Society, 41 boulevard de la République, 78401 CHATOU, France, email : frederique.berthereau@thales-avionics-es.com

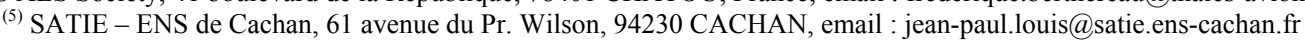

\begin{abstract}
Résumé-L'étude suivante présente un système de génération électrique travaillant à vitesse variable et fréquence fixe (VVFF) pour un réseau de bord d'avion. Ce système comprend une machine asynchrone à double alimentation (MADA) reliée par son stator au réseau de bord à générer. Elle est pilotée au rotor par l'intermédiaire d'un convertisseur statique permettant un fonctionnement en hypo et hyper synchronisme. Une machine synchrone à aimants permanents (MSAP) connectée à une turbine d'entraînement alimente ce convertisseur. La première partie de l'article présente la commande générale du système pour une simple charge linéaire RL. La MADA est commandée par une boucle interne de régulation du flux rotorique et une boucle externe de régulation de la tension du réseau. Nous présenterons également la commande de la MSAP et du bus continu du convertisseur. La deuxième partie de l'article développe la mise en place d'un filtre LC permettant de réduire les harmoniques induits par les charges non-linéaires, telles que les ponts redresseurs à six diodes. Les simulations effectuées sur MATLABSimulink prouvent l'efficacité du système proposé.
\end{abstract}

Mots-clés-Système à vitesse variable et fréquence fixe - réseau autonome - machine asynchrone à double alimentation - filtre LC - pont redresseur à 6 diodes

\section{INTRODUCTION}

De nos jours, deux systèmes utilisant des génératrices synchrones (GS) sont disponibles sur les réseaux de bord d'avions. Cette génération synchrone dite à 3 étages, consiste en une prise de mouvement sur le moteur de l'avion par une MSAP qui fournit une tension triphasée Celle-ci est redressée et sert à exciter par le stator une génératrice synchrone inversée qui fournit alors une tension triphasée par le rotor. Cette tension est à nouveau redressée

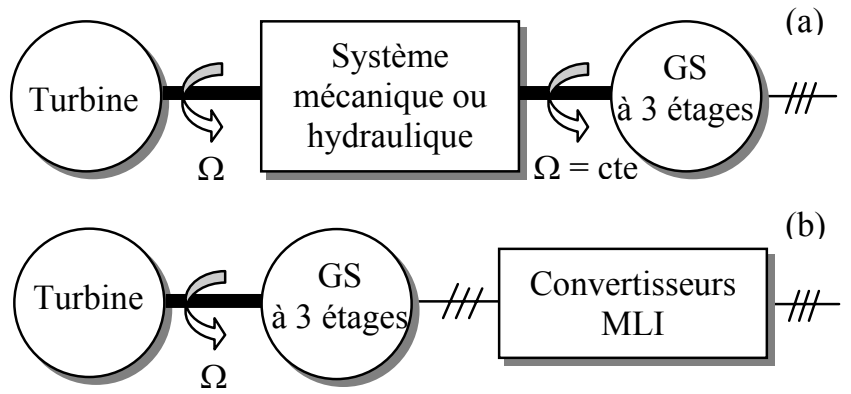

Fig. 1 Systèmes disponibles sur les réseaux de bord d'avion par un pont redresseur tournant placé sur l'axe du rotor et excite la troisième génératrice synchrone qui fournit ainsi par le stator une tension triphasée au réseau.

Le premier système basé sur une GS à 3 étages convertit la vitesse de rotation variable de la turbine en une vitesse de rotation constante par l'intermédiaire d'un système hydraulique ou mécanique (Fig. 1.a). La génératrice synchrone à trois étages est alors alimentée à vitesse fixe et fournit donc une fréquence fixe. L'inconvénient majeur de ce système est le coût nécessaire à sa maintenance. Le second système (Fig. 1.b) est un système où la génératrice synchrone à trois étages est placée juste après la turbine. La tension obtenue est donc à fréquence variable et attaque un convertisseur statique permettant l'obtention au final d'une tension à amplitude et fréquence fixes [15]. L'inconvénient de ce système réside en la nécessité de devoir dimensionner le convertisseur statique pour la pleine puissance du réseau.

D'un autre côté, les machines asynchrones à double alimentation sont de plus en plus étudiées en tant que sources de tension à fréquence fixe alors qu'elles sont entrânées à vitesse variable $[1,6,8]$. L'utilisation d'une MADA est attrayante quand la plage de variation de la vitesse de fonctionnement est limitée. En effet, dans ce cas, la puissance que doit fournir la cascade côté rotor n'est qu'une fraction de la puissance que la MADA doit fournir

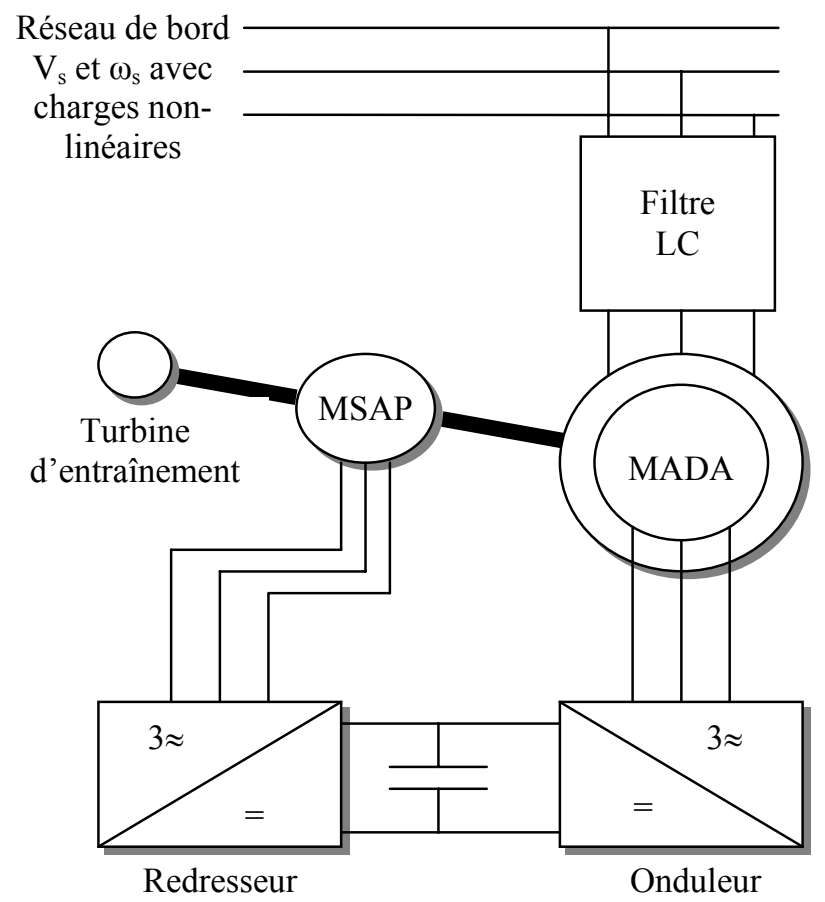

Fig. 2 Schéma global du système proposé 
au réseau $[1,6,8]$. De nos jours, beaucoup de recherches sont réalisées sur des éoliennes fournissant de l'énergie directement [9] ou de façon auxiliaire [4] à un réseau de puissance infinie par l'intermédiaire d'une MADA, cependant les études sur les réseaux autonomes [3] sont plus rares, vu la difficulté de contrôler des puissances souvent proches de la puissance nominale de la génératrice. Le fonctionnement d'une MADA alimentée par une génératrice Diesel a été étudiée dans [1] dans le cas d'un réseau de puissance infinie et dans [2] dans le cas d'un réseau autonome. Ces articles prouvent la faisabilité de ce type de systèmes. [10, 13] décrivent les caractéristiques et la commande d'un système basé sur deux MADA mises en cascade pour la génération en VVFF d'un réseau de bord d'avion. Cependant, à notre connaissance, aucun article ne traite la commande d'une MADA pilotée au rotor par un convertisseur statique pour ce type d'applications.

L'article suivant présente en premier lieu, la stratégie de commande d'une MADA de 50kVA, fournissant de l'énergie par le stator à un réseau autonome de bord d'avion de fréquence $400 \mathrm{~Hz}$ et de tension efficace par phase de $115 \mathrm{~V}$. Elle est pilotée par le rotor, par l'intermédiaire d'un convertisseur statique pour un fonctionnement en hypo et hyper synchronisme. Une machine synchrone à aimants permanents connectée à une turbine d'entraînement alimente ce convertisseur (Fig.2), qui est dimensionné proportionnellement à la plage de variation de la vitesse de rotation nécessaire $[1,2,6,12]$. Dans ce cas, $\mathrm{N}_{\mathrm{s}}$ étant la vitesse de synchronisme de $12000 \mathrm{tr} / \mathrm{mn}$ de la MADA, la vitesse varie de $0.6 \mathrm{~N}_{\mathrm{s}}$ en hyposynchronisme à $1.25 \mathrm{~N}_{\mathrm{s}}$ en hypersynchronisme. La MADA est contrôlée de manière à fournir une tension à amplitude et fréquence constantes indépendamment de la charge du réseau ou de la vitesse de la turbine d'entraînement. Cette commande est vectorielle dans un repère lié au champ statorique. Le flux statorique est aligné selon l'axe $d$ de ce repère permettant ainsi le découplage des puissances statoriques active et réactive de la MADA [5, 7]. La stratégie de commande est menée suivant deux boucles : une boucle interne de régulation du flux rotorique et une boucle externe permettant la régulation de la tension statorique suivant la valeur de consigne. De même, la MSAP est commandée par une boucle interne de courant et une boucle externe permettant la régulation de la tension du bus continu selon sa valeur nominale.

En second lieu, cet article traite l'introduction de charges non-linéaires telles que les ponts redresseurs à 6 diodes qui peuvent représenter jusqu'à $50 \%$ de la charge totale du réseau de bord. Ces charges introduisent des courants harmoniques importants et perturbent le bon fonctionnement du système. La solution proposée afin de diminuer ces harmoniques est la mise en place d'un filtre $\mathrm{LC}$ entre le stator et le réseau à fournir. Ce filtre, présenté figure 12 , permet l'obtention d'une tension de réseau quasisinusoïdale avec un taux de distorsion harmonique (TDH) inférieur à $5 \%$. De plus, ce filtre présente l'avantage d'une structure simple, d'un bon rendement, et d'une grande efficacité. Il est également très souvent utilisé pour les applications où de grandes puissances sont requises [14].

L'étude présente la modélisation et le principe de commande de la MADA dans la partie II, suivi de l'étude de la cascade côté rotor en III. La partie IV décrit les caractéristiques harmoniques d'un pont redresseur à 6 diodes et en $\mathrm{V}$, nous présentons la mise en place d'un filtre LC au stator de la MADA. Les résultats de simulation obtenus confirment la faisabilité du système proposé.

\section{MODELE ET PRINCIPE DE COMMANDE DE LA MACHINE SYNCHRONE A DOUBLE ALIMENTATION}

Les équations de la MADA dans un repère quelconque sont les suivantes [11]:

$$
\begin{aligned}
& \left\{\begin{array}{l}
\mathrm{v}_{\mathrm{sd}}=\mathrm{R}_{\mathrm{s}} \mathrm{i}_{\mathrm{sd}}+\frac{\mathrm{d}}{\mathrm{dt}} \varphi_{\mathrm{sd}}-\omega_{\mathrm{p}} \varphi_{\mathrm{sq}} \\
\mathrm{v}_{\mathrm{sq}}=\mathrm{R}_{\mathrm{s}} \mathrm{i}_{\mathrm{sq}}+\frac{\mathrm{d}}{\mathrm{dt}} \varphi_{\mathrm{sq}}+\omega_{\mathrm{p}} \varphi_{\mathrm{sd}}
\end{array}\right. \\
& \left\{\begin{array}{l}
\mathrm{v}_{\mathrm{rd}}=\mathrm{R}_{\mathrm{r}} \mathrm{i}_{\mathrm{rd}}+\frac{\mathrm{d}}{\mathrm{dt}} \varphi_{\mathrm{rd}}-\left(\omega_{\mathrm{p}}-\omega\right) \varphi_{\mathrm{rq}} \\
\mathrm{v}_{\mathrm{rq}}=\mathrm{R}_{\mathrm{r}} \mathrm{i}_{\mathrm{rq}}+\frac{\mathrm{d}}{\mathrm{dt}} \varphi_{\mathrm{rq}}+\left(\omega_{\mathrm{p}}-\omega\right) \varphi_{\mathrm{rd}}
\end{array}\right. \\
& \left\{\begin{array}{l}
\varphi_{\mathrm{sd}}=\mathrm{L}_{\mathrm{s}} \mathrm{i}_{\mathrm{sd}}+\mathrm{M}_{\mathrm{sr}} \mathrm{i}_{\mathrm{rd}} \\
\varphi_{\mathrm{sq}}=\mathrm{L}_{\mathrm{s}} \mathrm{i}_{\mathrm{sq}}+\mathrm{M}_{\mathrm{sr}} \mathrm{i}_{\mathrm{rq}}
\end{array}\right. \\
& \left\{\begin{array}{l}
\varphi_{\mathrm{rd}}=\mathrm{L}_{\mathrm{r}} \mathrm{i}_{\mathrm{rd}}+\mathrm{M}_{\mathrm{sr}} \mathrm{i}_{\mathrm{sd}} \\
\varphi_{\mathrm{rq}}=\mathrm{L}_{\mathrm{r}} \mathrm{i}_{\mathrm{rq}}+\mathrm{M}_{\mathrm{sr}} \mathrm{i}_{\mathrm{sq}}
\end{array}\right. \\
& \left\{\begin{array}{l}
\mathrm{P}_{\mathrm{s}}=\mathrm{v}_{\mathrm{sd}} \mathrm{i}_{\mathrm{sd}}+\mathrm{v}_{\mathrm{sq}} \mathrm{i}_{\mathrm{sq}} \\
\mathrm{Q}_{\mathrm{s}}=\mathrm{v}_{\mathrm{sq}} \mathrm{i}_{\mathrm{sd}}-\mathrm{v}_{\mathrm{sd}} \mathrm{i}_{\mathrm{sq}}
\end{array}\right.
\end{aligned}
$$

$\omega_{\mathrm{p}}$ est la vitesse de rotation du référentiel quelconque et $\omega$ la vitesse angulaire rotorique. $\mathrm{v}_{\mathrm{sd}}, \mathrm{v}_{\mathrm{sq}}, \mathrm{v}_{\mathrm{rd}}$ et $\mathrm{v}_{\mathrm{rq}}$ sont les composantes statoriques et rotoriques des tensions dans le repère de Park , $i_{\text {sd }}, i_{\text {sq }}, i_{\text {rd }}$ et $i_{\text {rq }}$ sont celles des courants, et $\varphi_{\mathrm{sd}}, \varphi_{\mathrm{sq}}, \varphi_{\mathrm{rd}}$ and $\varphi_{\mathrm{rq}}$, celles des flux. $\mathrm{R}_{\mathrm{s}}$ et $\mathrm{R}_{\mathrm{r}}$ sont les résistances statoriques et rotoriques, $\mathrm{L}_{\mathrm{S}}$ et $\mathrm{L}_{\mathrm{r}}$ les inductances propres et $\mathrm{M}_{\mathrm{sr}}$ l'inductance mutuelle. Finalement $\mathrm{P}_{\mathrm{s}}$ et $\mathrm{Q}_{\mathrm{s}}$ sont les puissances active et réactive de la MADA.

Le référentiel lié au champ statorique est choisi, tel que $\omega_{\mathrm{p}}=\omega_{\mathrm{s}}$. Le flux statorique est aligné avec l'axe $\mathrm{d}$ de ce référentiel ce qui correspond aux équations suivantes :

$$
\varphi_{\mathrm{sq}}=0 \quad \text { et } \quad \frac{\mathrm{d}}{\mathrm{dt}} \varphi_{\mathrm{sq}}=0
$$

Pour pouvoir présenter les principes de cette commande, nous négligeons les résistances statoriques et supposons que l'état permanent est atteint. La tension du réseau de bord est donc fixe en amplitude et en fréquence comme dans le cas des réseaux infinis, d'où :

$$
\begin{aligned}
& \frac{\mathrm{d}}{\mathrm{dt}} \varphi_{\mathrm{sd}}=0 \\
& \text { et } \quad\left\{\begin{array}{l}
\mathrm{v}_{\mathrm{sd}} \approx 0 \\
\mathrm{v}_{\mathrm{sq}} \approx \omega_{\mathrm{s}} \varphi_{\mathrm{sd}}
\end{array}\right.
\end{aligned}
$$

D'après $(3,4,6), \sigma$ étant le coefficient de dispersion de la MADA, la contrainte (9) correspond à la bonne orientation du repère choisi $[3,4,11]$ :

$$
\mathrm{i}_{\mathrm{rq}}=-\frac{\mathrm{L}_{\mathrm{s}}}{\mathrm{M}_{\mathrm{sr}}} \mathrm{i}_{\mathrm{sq}} \Leftrightarrow \varphi_{\mathrm{rq}}=-\frac{\sigma \mathrm{L}_{\mathrm{s}} \mathrm{L}_{\mathrm{r}}}{\mathrm{M}_{\mathrm{sr}}} \mathrm{i}_{\mathrm{sq}}
$$




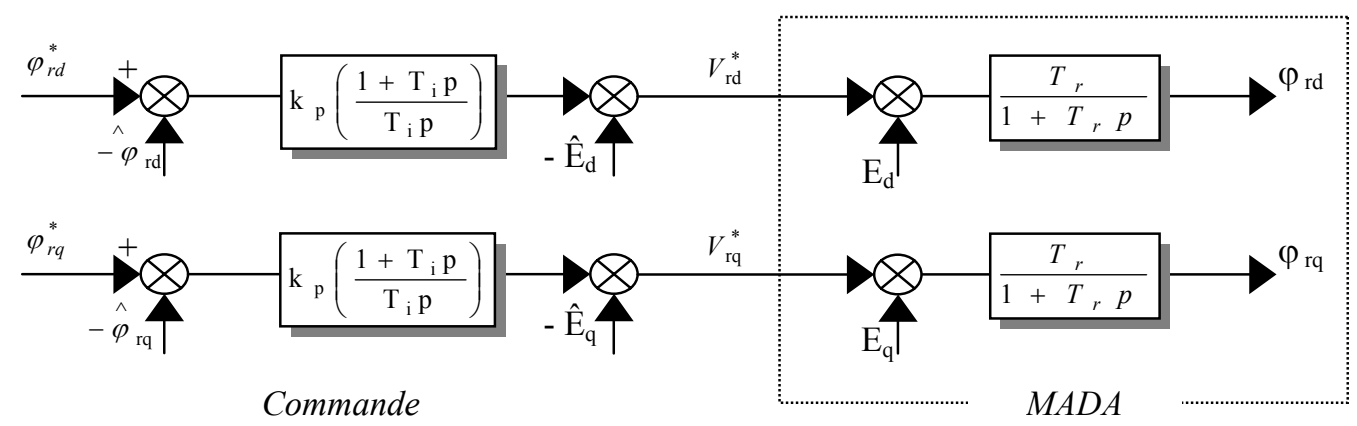

Fig. 3 Schéma-bloc de la régulation du flux rotorique de la MADA

D'après $(5,8,9)$, les puissances statoriques active et réactive de la MADA deviennent :

$$
\begin{aligned}
& \mathrm{P}_{\mathrm{s}}=\frac{(1-\sigma)}{\sigma \mathrm{M}_{\mathrm{sr}}} \sqrt{3} \mathrm{~V}_{\mathrm{s}} \varphi_{\mathrm{rq}} \\
& \mathrm{Q}_{\mathrm{s}}=\frac{(1-\sigma)}{\sigma \mathrm{M}_{\mathrm{sr}}}\left(\sqrt{3} \mathrm{~V}_{\mathrm{s}} \varphi_{\mathrm{rd}}-\frac{\mathrm{L}_{\mathrm{r}}}{\mathrm{M}_{\mathrm{sr}}} \frac{3 \mathrm{~V}_{\mathrm{s}}^{2}}{\omega_{\mathrm{s}}}\right)
\end{aligned}
$$

(10) montre le découplage des puissances active via $\varphi_{\mathrm{rq}}$ et réactive via $\varphi_{\text {rd }}$ lorsque le régime permanent est atteint.

\section{A. Modèle d'état de la MADA}

En mode générateur, la MADA est représentée par le système d'état à temps variant suivant :

$$
\begin{aligned}
& {\left[\dot{\Phi}_{\mathrm{r}}\right]=[\mathrm{A}]\left[\Phi_{\mathrm{r}}\right]+[\mathrm{B}]\left[\mathrm{V}_{\mathrm{r}}\right]+[\mathrm{E}]\left[\mathrm{I}_{\mathrm{s}}\right]} \\
& {\left[\mathrm{V}_{\mathrm{s}}\right]=[\mathrm{C}]\left[\Phi_{\mathrm{r}}\right]+[\mathrm{D}]\left[\mathrm{V}_{\mathrm{r}}\right]+[\mathrm{F}]\left[\mathrm{I}_{\mathrm{s}}\right]+[\mathrm{G}]\left[\dot{\mathrm{I}}_{\mathrm{S}}\right.}
\end{aligned}
$$

avec :

$$
\begin{aligned}
& {\left[\mathrm{V}_{\mathrm{s}}\right]=\left[\begin{array}{c}
\mathrm{v}_{\mathrm{sd}} \\
\mathrm{v}_{\mathrm{sq}}
\end{array}\right] ;\left[\Phi_{\mathrm{r}}\right]=\left[\begin{array}{c}
\varphi_{\mathrm{rd}} \\
\varphi_{\mathrm{rq}}
\end{array}\right] ;\left[\mathrm{V}_{\mathrm{r}}\right]=\left[\begin{array}{c}
\mathrm{v}_{\mathrm{rd}} \\
\mathrm{v}_{\mathrm{rq}}
\end{array}\right] ;\left[\mathrm{I}_{\mathrm{s}}\right]=\left[\begin{array}{c}
\mathrm{i}_{\mathrm{sd}} \\
\mathrm{i}_{\mathrm{sq}}
\end{array}\right]} \\
& {[\mathrm{A}]=\left[\begin{array}{cc}
-\frac{\mathrm{R}_{\mathrm{r}}}{\mathrm{L}_{\mathrm{r}}} & \omega_{\mathrm{r}} \\
-\omega_{\mathrm{r}} & -\frac{\mathrm{R}_{\mathrm{r}}}{\mathrm{L}_{\mathrm{r}}}
\end{array}\right] ;[\mathrm{B}]=\left[I_{2}\right] ;[\mathrm{E}]=\frac{\mathrm{R}_{\mathrm{r}} \mathrm{M}_{\mathrm{sr}}}{\mathrm{L}_{\mathrm{r}}}\left[I_{2}\right]} \\
& {[\mathrm{C}]=-\frac{\mathrm{M}_{\mathrm{sr}}}{\mathrm{L}_{\mathrm{r}}}\left[\begin{array}{cc}
\frac{\mathrm{R}_{\mathrm{r}}}{\mathrm{L}_{\mathrm{r}}} & \omega \\
-\omega & \frac{\mathrm{R}_{\mathrm{r}}}{\mathrm{L}_{\mathrm{r}}}
\end{array}\right] ;[\mathrm{D}]=\frac{\mathrm{M}_{\mathrm{sr}}}{\mathrm{L}_{\mathrm{r}}}\left[I_{2}\right] ;}
\end{aligned}
$$$$
[F]=\left[\begin{array}{cc}
\mathrm{R}_{\mathrm{s}}+\frac{\mathrm{M}_{\mathrm{sr}}^{2}}{\mathrm{~L}_{\mathrm{r}}^{2}} \mathrm{R}_{\mathrm{r}} & -\sigma \mathrm{L}_{\mathrm{s}} \omega_{\mathrm{s}} \\
\sigma \mathrm{L}_{\mathrm{s}} \omega_{\mathrm{s}} & \mathrm{R}_{\mathrm{s}}+\frac{\mathrm{M}_{\mathrm{sr}}^{2}}{\mathrm{~L}_{\mathrm{r}}^{2}} \mathrm{R}_{\mathrm{r}}
\end{array}\right] ; \quad\left[\begin{array}{c}
{\left[I_{2}\right]=\left[\begin{array}{cc}
1 & 0 \\
0 & 1
\end{array}\right]} \\
{[\mathrm{G}]=\sigma \mathrm{L}_{\mathrm{s}}\left[I_{2}\right]}
\end{array}\right.
$$

$\left[\mathrm{V}_{\mathrm{r}}\right],\left[\mathrm{V}_{\mathrm{s}}\right]$ et $\left[\Phi_{\mathrm{r}}\right]$ sont respectivement les vecteurs d'entrée, de sortie et d'état du système. Etant donné que $\left[\mathrm{I}_{\mathrm{s}}\right]$ dépend de la charge, ce vecteur est considéré comme une perturbation.

Dans le cas d'une MADA fonctionnant en génératrice, la difficulté vient des termes dérivés de la perturbation (12), qui sont difficiles à simuler. De plus, il existe un lien direct entre l'entrée et la sortie du système.

L'originalité de cette nouvelle méthode de commande vient du choix du flux rotorique comme vecteur de contrôle, en effet (11) montre que le flux rotorique est le vecteur d'état naturel de la MADA et de plus, il permet un contrôle direct sur la tension rotorique de celle-ci. Comparée à une régulation en courant, cette méthode permet une minimisation des harmoniques introduits par des charges non-linéaires.

\section{B. Boucle interne de régulation du flux rotorique}

A partir de (11), on peut déduire le système suivant où $E_{d}$ et $E_{q}$ sont des termes de couplage :

$$
\left\{\begin{array}{l}
\frac{\mathrm{d}}{\mathrm{dt}} \varphi_{\mathrm{rd}}=\mathrm{v}_{\mathrm{rd}}-\frac{1}{\mathrm{~T}_{\mathrm{r}}} \varphi_{\mathrm{rd}}+\mathrm{E}_{\mathrm{d}} \\
\frac{\mathrm{d}}{\mathrm{dt}} \varphi_{\mathrm{rq}}=\mathrm{v}_{\mathrm{rq}}-\frac{1}{\mathrm{~T}_{\mathrm{r}}} \varphi_{\mathrm{rq}}+\mathrm{E}_{\mathrm{q}}
\end{array}\right.
$$

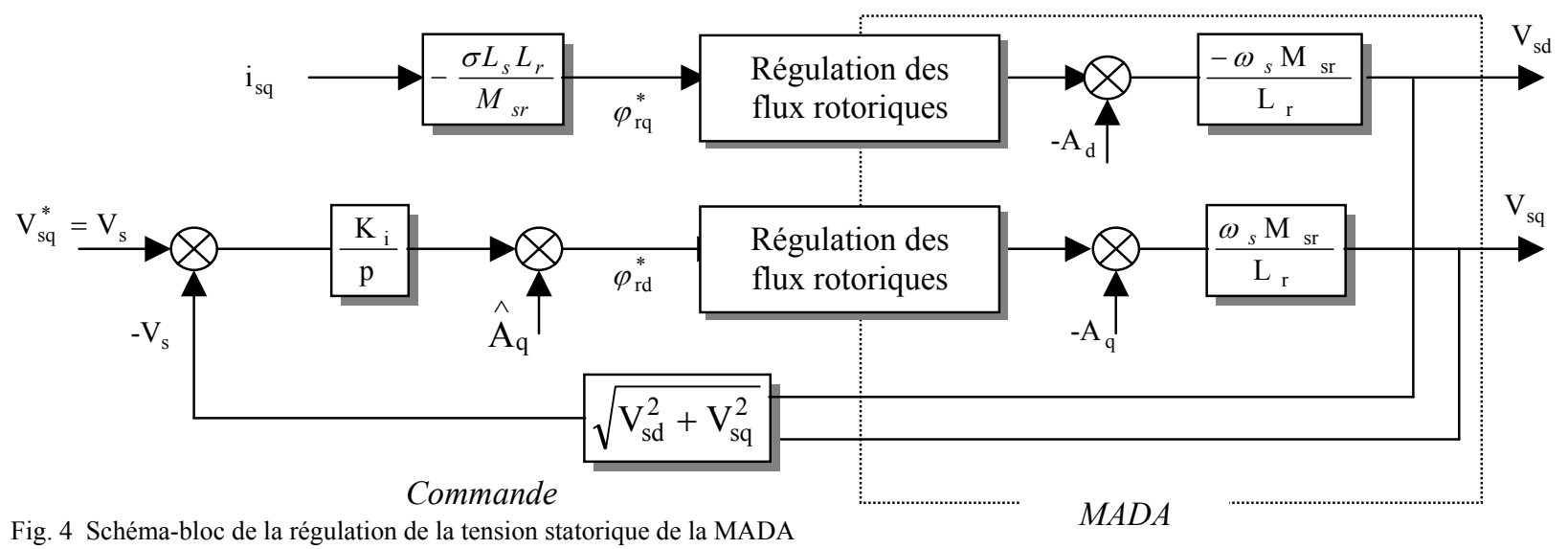




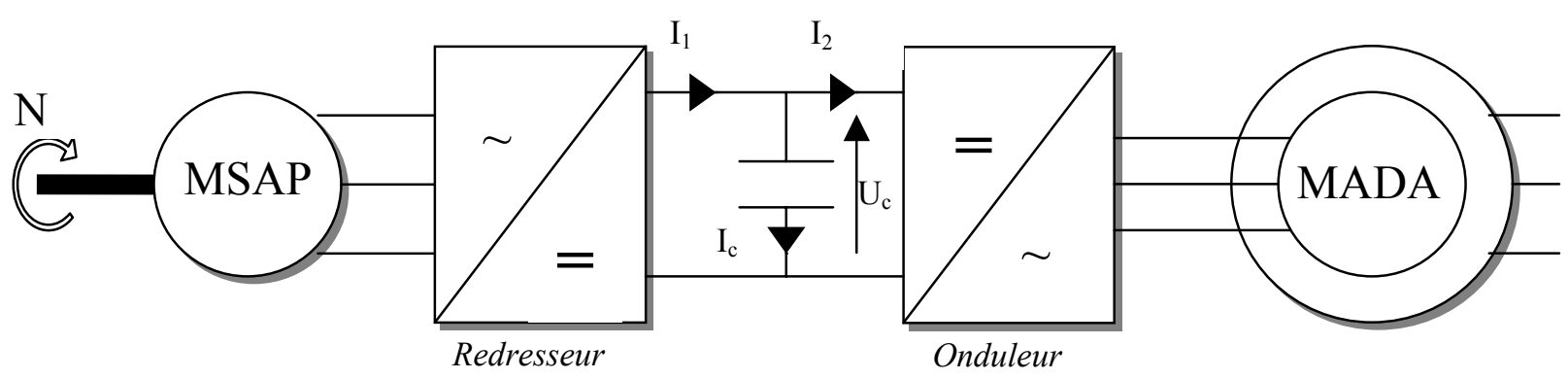

Fig. 5 Schéma global de la cascade côté rotor

$$
\left\{\begin{array}{l}
\mathrm{E}_{\mathrm{d}}=\frac{\mathrm{M}_{\mathrm{sr}}}{\mathrm{T}_{\mathrm{r}}} \mathrm{i}_{\mathrm{sd}}+\omega_{\mathrm{r}} \varphi_{\mathrm{rq}} \\
\mathrm{E}_{\mathrm{q}}=\frac{\mathrm{M}_{\mathrm{sr}}}{\mathrm{T}_{\mathrm{r}}} \mathrm{i}_{\mathrm{sq}}-\omega_{\mathrm{r}} \varphi_{\mathrm{rq}}
\end{array}\right.
$$

Les fonctions de transfert entre les flux et les tensions rotoriques ne dépendent que de la constante de temps rotorique $T_{r}$. La régulation peut donc être réalisée avec de simples correcteurs PI. De plus, les termes de couplage peuvent être compensés comme présenté sur le schéma-bloc de la figure 3.

\section{Boucle de régulation de la tension statorique}

On a déjà vu dans la partie II, que la contrainte (9) correspond à l'orientation correcte du référentiel choisi. L'amplitude de la tension statorique est donnée par :

$$
\left|\mathrm{V}_{\mathrm{s}}\right|=\sqrt{\mathrm{V}_{\mathrm{sd}}^{2}+\mathrm{V}_{\mathrm{sq}}^{2}}
$$

Celle-ci est contrôlée par une boucle externe (Fig. 4), puisque (12) montre que lorsque la MADA fonctionne en génératrice sur un réseau autonome, la tension statorique est le vecteur de sortie du système. Les équations $(8,12,15)$ permettent d'obtenir le système suivant, où $A_{d}$ et $A_{q}$ sont des termes de perturbation que l'on peut compenser. Les fonctions de transfert entre les tensions statoriques et les flux rotoriques sont de simples gains, des intégrateurs permettront d'annuler l'erreur statique entre les tensions mesurée et désirée.

$$
\begin{aligned}
& \left\{\begin{array}{l}
\left|\mathrm{V}_{\mathrm{s}}\right| \approx \mathrm{V}_{\mathrm{sq}} \\
\mathrm{v}_{\mathrm{sd}}=-\omega_{\mathrm{s}} \frac{\mathrm{M}_{\mathrm{sr}}}{\mathrm{L}_{\mathrm{r}}}\left(\varphi_{\mathrm{rq}}+\mathrm{A}_{\mathrm{d}}\right) \\
\mathrm{v}_{\mathrm{sq}}=\omega_{\mathrm{s}} \frac{\mathrm{M}_{\mathrm{sr}}}{\mathrm{L}_{\mathrm{r}}}\left(\varphi_{\mathrm{rd}}+\mathrm{A}_{\mathrm{q}}\right)
\end{array}\right. \\
& \left\{\begin{array}{l}
\mathrm{A}_{\mathrm{d}}=\frac{\mathrm{L}_{\mathrm{r}}}{\mathrm{M}_{\mathrm{sr}}}\left(-\frac{\mathrm{R}_{\mathrm{s}}}{\omega_{\mathrm{s}}} \mathrm{i}_{\mathrm{sd}}+\sigma \mathrm{L}_{\mathrm{s}} \mathrm{i}_{\mathrm{sq}}\right) \\
\mathrm{A}_{\mathrm{q}}=\frac{\mathrm{L}_{\mathrm{r}}}{\mathrm{M}_{\mathrm{sr}}}\left(\frac{\mathrm{R}_{\mathrm{s}}}{\omega_{\mathrm{s}}} \mathrm{i}_{\mathrm{sq}}+\sigma \mathrm{L}_{\mathrm{s}} \mathrm{i}_{\mathrm{sd}}\right)
\end{array}\right.
\end{aligned}
$$

\section{CASCADE COTE Rotor}

La figure 5 présente le système vu du côté rotor de la MADA. Cette configuration utilisant un convertisseur

statique est très fréquente pour les applications à haute puissance et à plage de variation de la vitesse limitée. Cette méthode permet un fonctionnement en dessous, au-dessus et à la vitesse de synchronisme. Ce sont les limites de cette plage de variation de la vitesse qui fixent la puissance du convertisseur.

La commande de la MADA a été décrite dans la partie II et la suite présentera la commande de la MSAP. L'objectif de cette commande est de garder la tension du bus continu constante indépendamment de la puissance rotorique. Ceci sera réalisé suivant deux boucles de régulation : une boucle interne de régulation du courant statorique de la MSAP et une boucle externe régulant la tension du bus continu à sa valeur nominale $\left(\mathrm{U}_{\mathrm{c}}=350 \mathrm{~V}\right)$.

\section{A. Régulation du courant statorique de la MSAP}

Le modèle de la MSAP dans un repère lié au synchronisme est donné par le système (19). $\mathrm{E}_{\mathrm{md}}$ et $\mathrm{E}_{\mathrm{mq}}$ sont des termes de couplage. $R_{m s}$ est la résistance statorique, $L_{d}$ et $\mathrm{L}_{\mathrm{q}}$ sont les inductances directes et en quadrature. $\mathrm{V}_{\mathrm{d}}, \mathrm{V}_{\mathrm{q}}$, $\mathrm{I}_{\mathrm{d}}$ et $\mathrm{L}_{\mathrm{q}}$ sont les composantes dq des tensions et courants statoriques. $\varphi_{\mathrm{f}}$ est le flux de l'aimant permanent.

$$
\begin{aligned}
& \left\{\begin{array}{l}
V_{d}=R_{m s} I_{d}+L_{d} \frac{d}{d t} I_{d}-E_{m d} \\
V_{q}=R_{m s} I_{q}+L_{q} \frac{d}{d t} I_{q}-E_{m q}
\end{array}\right. \\
& \left\{\begin{array}{l}
E_{m d}=\omega L_{q} I_{q} \\
E_{m q}=-\omega L_{d} I_{d}-\omega \varphi_{f}
\end{array}\right.
\end{aligned}
$$

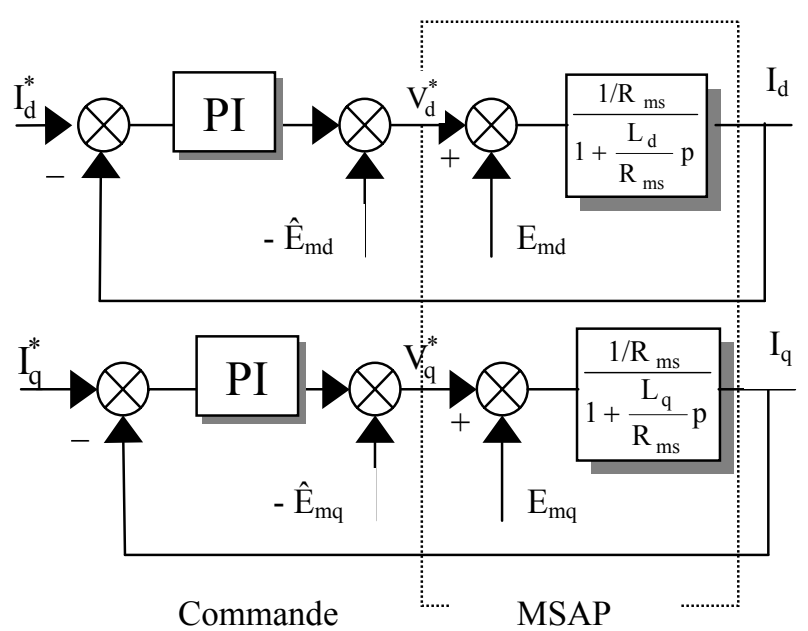

Fig. 6 Boucle de régulation du courant de la MSAP 
Les fonctions de transfert entre les tensions et les courants de la MSAP sont du premier ordre et sont régulées par des correcteurs PI comme le montre le schéma-bloc de la figure 6 .

\section{B. Courant de référence suivant l'axe d}

En régime permanent et en négligeant les résistances statoriques, $(19,20)$ donnent le système suivant :

$$
\left\{\begin{array}{l}
\mathrm{V}_{\mathrm{d}}=-\omega \mathrm{L}_{\mathrm{q}} \mathrm{I}_{\mathrm{q}} \\
\mathrm{V}_{\mathrm{q}}=\omega \mathrm{L}_{\mathrm{d}} \mathrm{I}_{\mathrm{d}}+\omega \varphi_{\mathrm{f}}
\end{array}\right.
$$

De plus, en négligeant les pertes introduites par le convertisseur, on peut écrire la relation (22) où $\mathrm{P}$ est la puissance active, $\mathrm{U}_{\mathrm{c}}$ la tension $\mathrm{du}$ bus continu et $\mathrm{I}_{1}$ le courant de sortie du redresseur.

$$
\mathrm{P}=\mathrm{V}_{\mathrm{d}} \mathrm{I}_{\mathrm{d}}+\mathrm{V}_{\mathrm{q}} \mathrm{I}_{\mathrm{q}}=\mathrm{U}_{\mathrm{c}} \mathrm{I}_{1}
$$
tire :

D'après $(21,22)$ et sachant que dans notre cas $\mathrm{L}_{\mathrm{d}}=\mathrm{L}_{\mathrm{q}}$, on

$$
\begin{aligned}
& \mathrm{I}_{\mathrm{q}}^{*}=\frac{\mathrm{P}}{\omega \varphi_{\mathrm{f}}} \\
& \mathrm{V}_{\mathrm{d}}=\frac{\mathrm{P} \mathrm{L_{ \textrm {q } }}}{\varphi_{\mathrm{f}}}
\end{aligned}
$$

$(23,24)$ montrent que les composantes directe de la tension et en quadrature du courant dépendent de la puissance rotorique désirée. Une méthode de commande classique de la MSAP cherche à obtenir le maximum de puissance pour un minimum de courant. Cependant si $\mathrm{I}_{\mathrm{d}}^{*}=0$, la tension statorique est donnée par (25). Cette tension est acceptable tant qu'elle est inférieure à la tension limite $\mathrm{V}_{\text {lim }}$ fixée par la tension du bus continu (26). Sinon, la référence suivant l'axe d du courant est donnée par (27) et sert à défluxer la MSAP. De cette manière la tension limite n'est pas dépassée.

$$
\begin{aligned}
& |\mathrm{V}|=\sqrt{\left(\frac{\mathrm{PL}_{\mathrm{q}}}{\varphi_{\mathrm{f}}}\right)^{2}+\left(\mathrm{w} \varphi_{\mathrm{f}}\right)^{2}} \\
& |\mathrm{~V}|<\mathrm{V}_{\text {lim }}
\end{aligned}
$$

$$
\mathrm{I}_{\mathrm{d} 1}^{*}=\frac{\left(\sqrt{\mathrm{V}_{\mathrm{lim}}^{2}-\mathrm{V}_{\mathrm{q}}{ }^{2}}-\omega \varphi_{\mathrm{f}}\right)}{\omega \mathrm{L}_{\mathrm{d}}}
$$

Finalement, la stratégie de commande se fera suivant :

$$
\left\{\begin{array}{lll}
|\mathrm{V}|>\mathrm{V}_{\lim } & \Rightarrow & \mathrm{I}_{\mathrm{d}}^{*}=\mathrm{I}_{\mathrm{d} 1}^{*} \\
|\mathrm{~V}|<\mathrm{V}_{\lim } & \Rightarrow & \mathrm{I}_{\mathrm{d}}^{*}=0
\end{array}\right.
$$

\section{Boucle de régulation de la tension continue}

D'après $(22,23)$, l'équation des puissances devient :

$$
\mathrm{P}=\mathrm{U}_{\mathrm{c}} \mathrm{I}_{1}=\omega \varphi_{\mathrm{f}} \mathrm{I}_{\mathrm{q}} \Rightarrow \mathrm{I}_{\mathrm{q}}=\frac{\mathrm{U}_{\mathrm{c}}}{\omega \varphi_{\mathrm{f}}} \mathrm{I}_{1}
$$

Le schéma-bloc de cette boucle est donné figure 7. La régulation se fait avec des correcteurs PI après avoir fixé le facteur d'amortissement et la fréquence propre désirés.

Le système proposé a été testé sous MATLAB-Simulink de la limite inférieure à la limite supérieure de vitesse de fonctionnement. La convention de signe adoptée pour la MADA, est celle d'un fonctionnement moteur en mode hypersynchrone.

Les résultats de simulation obtenus prouvent la faisabilité du système proposé pour la charge maximale de $50 \mathrm{kVA}$ avec un facteur de puissance de 1 à $0.1 \mathrm{~s}$ et de 0.8 à $0.25 \mathrm{~s}$., suivi d'une variation de vitesse de la limite inférieure à la limite supérieure à $0.4 \mathrm{~s}$.

La figure 8 présente les puissances active et réactive $P_{s}$ et $\mathrm{Q}_{\mathrm{s}}$ (b) de la MADA, les puissances mécanique et rotorique $\mathrm{P}_{\mathrm{m}}$ et $\mathrm{P}_{\mathrm{r}}(\mathrm{c})$, l'amplitude des tensions statorique et rotorique $\mathrm{V}_{\mathrm{s}}$ et $\mathrm{V}_{\mathrm{r}}(\mathrm{d})$, la tension aux bornes de la capacité $\mathrm{du}$ bus continu (e) et la tension d'une phase statorique (f), durant une variation de vitesse de la limite inférieure $0.6 \mathrm{~N}_{\mathrm{s}}$ à la limite supérieure $1.25 \mathrm{~N}_{\mathrm{s}}(\mathrm{a})$. Les valeurs obtenues montrent l'atténuation de l'amplitude et de la fréquence de la tension rotorique lors du passage de la vitesse de l'hypo à l'hypersynchronisme. La tension statorique $\mathrm{V}_{\mathrm{s}}$ et la tension continue $\mathrm{U}_{\mathrm{c}}$ sont tout à fait contrôlées que ce soit durant les variations de vitesse ou de charge. Ceci prouve donc la faisabilité du système en hypo et hypersynchronisme.

Cependant, nous verrons par la suite que l'introduction de charges non-linéaires perturbent le système et donnent lieu à des résultats inacceptables à cause des courants harmoniques induits.

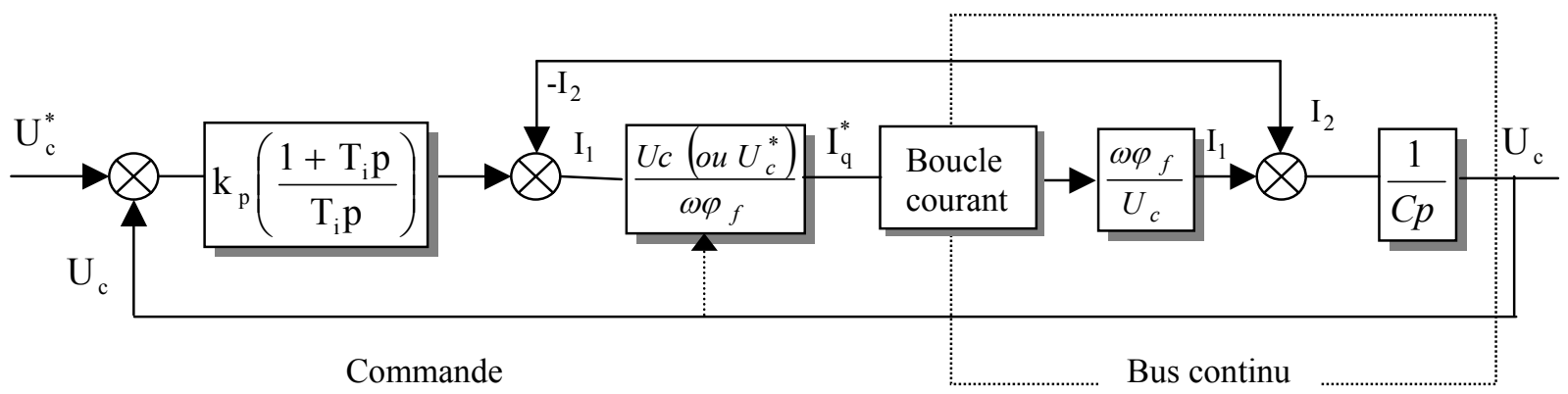

Fig. 7 Régulation de la tension du bus continu 

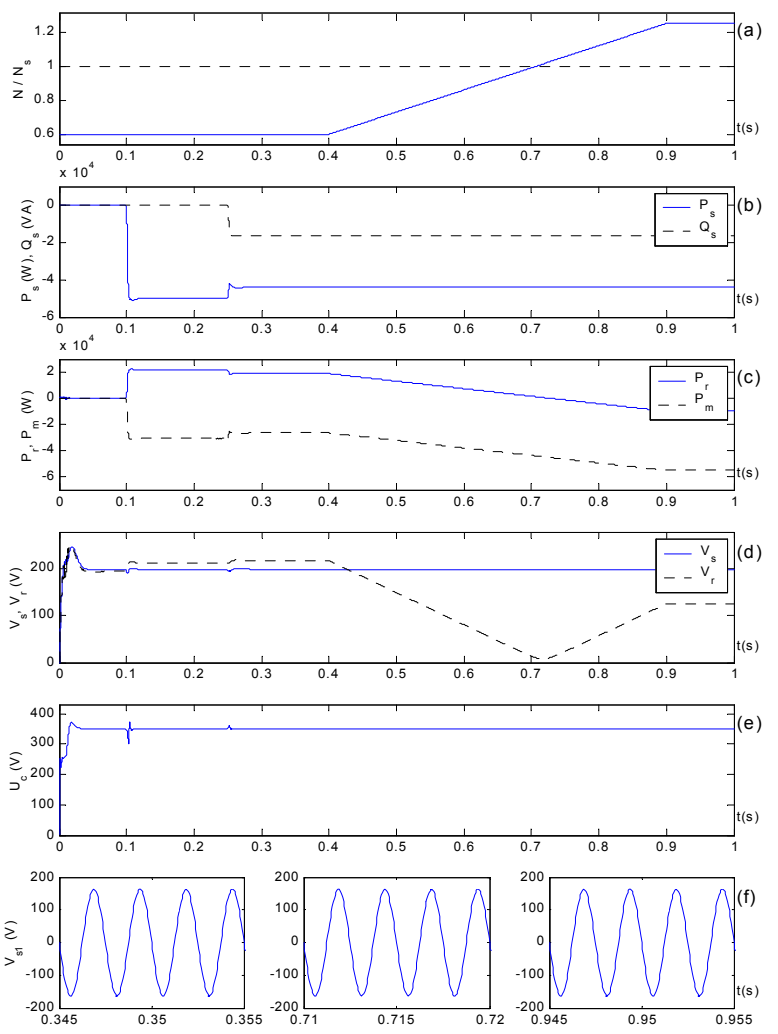

Fig. 8 Variation de charge suivie d'une variation de vitesse de 0.6 $\mathrm{N}_{\mathrm{s}}$ à $1.25 \mathrm{~N}_{\mathrm{s}}$ avec une charge de $50 \mathrm{kVA}$ et $\cos \varphi=0.8$

\section{CARACTERISTIQUES HARMONIQUES D'UN PONT REDRESSEUR A 6 DIODES}

Sur un réseau de bord d'avion, les charges non-linéaires sont essentiellement dues à la présence de ponts redresseurs à six diodes (Fig. 9). En effet, ce type de convertisseurs induit un grand nombre d'harmoniques de courant :

$$
\begin{aligned}
& \mathrm{I}_{\mathrm{sf}}=\frac{\sqrt{3}}{\pi} \mathrm{I}_{\mathrm{c}} \\
& \mathrm{I}_{\mathrm{sh}}=\frac{\mathrm{I}_{\mathrm{sf}}}{\mathrm{h}} \quad, \quad \mathrm{h}=6 \mathrm{n} \pm 1 \quad, \mathrm{n} \geq 1 \\
& \mathrm{THD}_{\mathrm{Is}}=\frac{\sqrt{\sum \mathrm{I}_{\mathrm{sh}}^{2}}}{\mathrm{I}_{\mathrm{sf}}} \approx 30 \%
\end{aligned}
$$

$\mathrm{I}_{\mathrm{sf}}$ représente l'amplitude du courant fondamental, $\mathrm{I}_{\mathrm{sh}}$ celle du courant harmonique de rang $h$ et $I_{c}$ le courant continu traversant la résistance de charge. $\mathrm{TDH}_{\mathrm{Is}}$ est le taux de distorsion harmonique du courant de charge. Les principaux harmoniques à éliminer sont de rang 5, 7, 11 et 13.

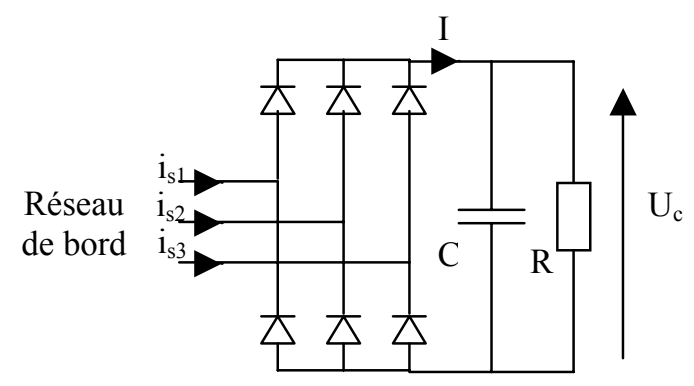

Fig. 9 Structure d'un pont redresseur à six diodes
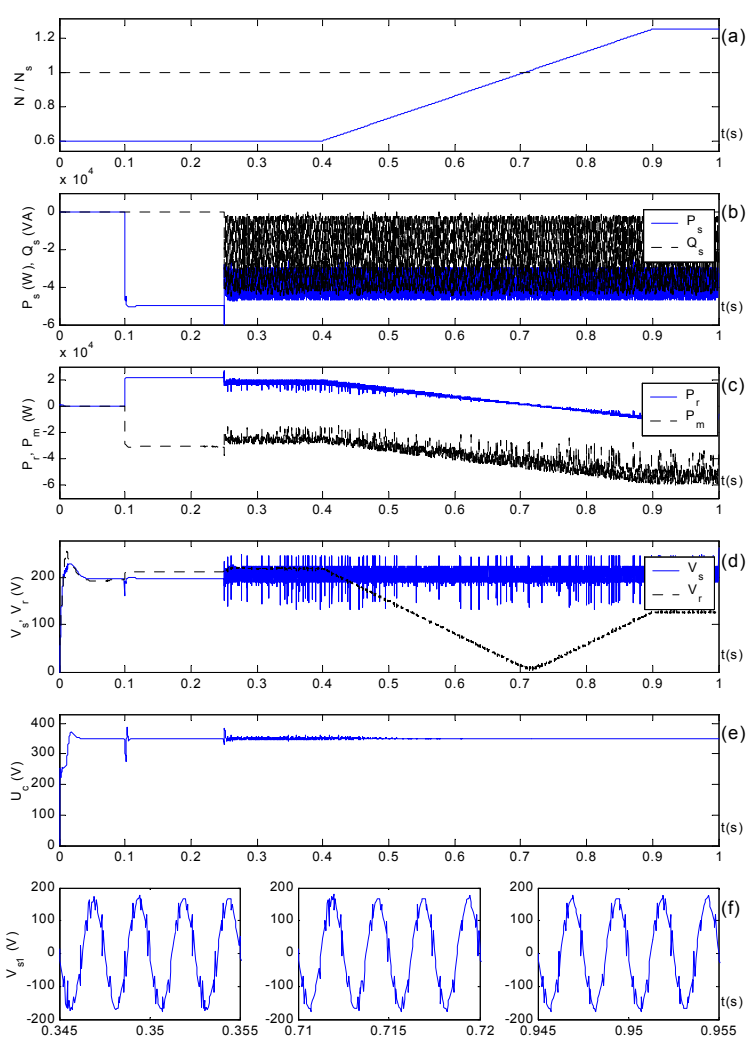

Fig. 10 Variation de charge NL à 50\% suivie d'un changement de vitesse de $0.6 \mathrm{~N}_{\mathrm{s}}$ à $1.25 \mathrm{~N}_{\mathrm{s}}$ avec $50 \mathrm{kVA}$ de charge

L'insertion d'un pont redresseur à six diodes dans le système ne change pas la procédure de test, la seule différence consiste dans l'introduction de charges nonlinéaires égales à $50 \%$ de la charge totale à $0.25 \mathrm{~s}$. Les résultats, présentés à la figure 10, montrent clairement la détérioration de la tension du stator, causée par l'introduction de ces 50\% de charges non-linéaires (NL). Le TDH de la tension de réseau est alors de 12\% (Fig. 11a), alors que notre cahier des charges impose un TDH de 5\%. Les puissances rotoriques et mécaniques sont aussi détériorées par cette charge, mais c'est surtout les puissances active et réactive du stator qui présentent d'importantes fluctuations dues aux harmoniques induits par le pont redresseur à 6 diodes. Ces harmoniques augmentent les pertes dans la MADA et favorisent l'échauffement excessif de celle-ci [14].

La figure 11 montre que le TDH de la tension statorique
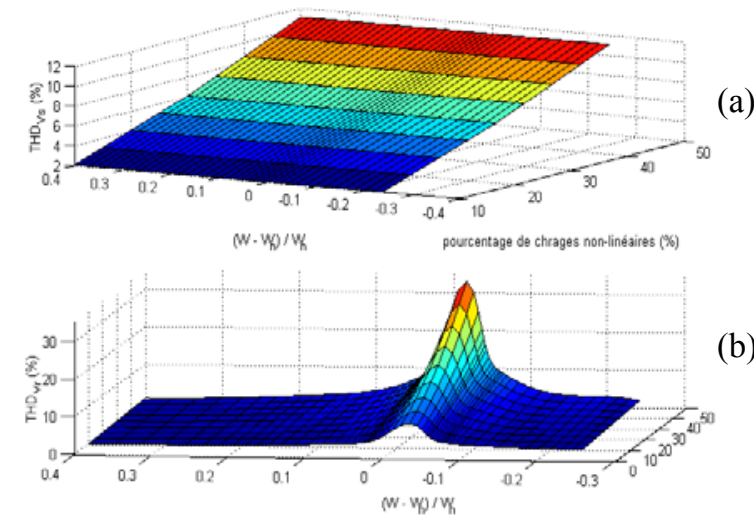

Fig. 11 Taux de distorsion harmonique des tensions statoriques (a) et rotoriques (b) 
ne dépend que du pourcentage de charges non-linéaires sur le réseau, tandis que le TDH de la tension rotorique dépend aussi de la vitesse de fonctionnement du système. Il devient plus important lorsque la vitesse du système se rapproche de la vitesse de synchronisme du fait que l'amplitude de $\mathrm{V}_{\mathrm{r}}$ tend vers zéro. (13) et (14) montrent que les termes de couplage dépendent de $\omega_{\mathrm{r}}$ et $\mathrm{I}_{\mathrm{s}}$, donc, près de la vitesse de synchronisme, $I_{s}$, qui dépend de la charge, est plus importante que $\omega_{\mathrm{r}}$, qui est proche de zéro, d'où l'effet des harmoniques sur la tension rotorique.

\section{Mise EN PLACE D'UN FILTRE LC}

\section{A. Caractéristiques du filtre $L C$}

Le filtre LC est placé du côté du stator de la MADA afin d'éliminer les harmoniques de tension du réseau de bord. Le modèle équivalent monophasé du filtre est donné à la figure 12 , où $\mathrm{L}$ et $\mathrm{r}$ représentent l'inductance de filtrage et sa résistance interne, et $\mathrm{C}$ la capacité du filtre. Celui-ci peut être représenté par les équations d'état suivantes :

$$
\frac{\mathrm{d}}{\mathrm{dt}}\left[\begin{array}{c}
\mathrm{I}_{\mathrm{s}} \\
\mathrm{V}_{\mathrm{g}}
\end{array}\right]=\left[\begin{array}{cc}
-\frac{\mathrm{r}}{\mathrm{L}} & -\frac{1}{\mathrm{~L}} \\
\frac{1}{\mathrm{C}} & 0
\end{array}\right]\left[\begin{array}{c}
\mathrm{I}_{\mathrm{s}} \\
\mathrm{V}_{\mathrm{g}}
\end{array}\right]+\left[\begin{array}{cc}
0 & \frac{1}{\mathrm{~L}} \\
-\frac{1}{\mathrm{C}} & 0
\end{array}\right]\left[\begin{array}{c}
\mathrm{I}_{\mathrm{g}} \\
\mathrm{V}_{\mathrm{s}}
\end{array}\right]
$$

$\mathrm{V}_{\mathrm{s}}, \mathrm{I}_{\mathrm{s}}, \mathrm{I}_{\mathrm{g}}, \mathrm{V}_{\mathrm{g}}$ sont respectivement la tension et le courant statoriques de la MADA, et le courant et la tension du réseau de bord. $\mathrm{V}_{\mathrm{s}}$ peut être considérée comme la variable d'entrée du filtre, $I_{g}$ la variable de perturbation et $V_{g}$ la variable de sortie du filtre. A partir de (33), on peut écrire quatre fonctions de transfert pour décrire le fonctionnement du filtre :

$$
\left[\begin{array}{c}
\mathrm{I}_{\mathrm{s}}(\mathrm{s}) \\
\mathrm{V}_{\mathrm{g}}(\mathrm{s})
\end{array}\right]=\frac{\omega_{\mathrm{o}}^{2}}{\mathrm{~s}^{2}+2 \mathrm{~m} \omega_{\mathrm{o}} \mathrm{s}+\omega_{\mathrm{o}}^{2}}\left[\begin{array}{cc}
1 & \mathrm{Cs} \\
\mathrm{Ls}+\mathrm{r} & 1
\end{array}\right]\left[\begin{array}{c}
\mathrm{I}_{\mathrm{g}}(\mathrm{s}) \\
\mathrm{V}_{\mathrm{s}}(\mathrm{s})
\end{array}\right]
$$

$$
\begin{aligned}
& \text { avec } \quad \omega_{0}=\frac{1}{\sqrt{\mathrm{LC}}} \text { la pulsation propre, } \\
& \text { et } \quad \mathrm{m}=\frac{\mathrm{r}}{2 \sqrt{\frac{\mathrm{L}}{\mathrm{C}}}} \quad \text { le coefficient d'amortissement. }
\end{aligned}
$$

On peut ainsi remarquer que les harmoniques de courant du réseau dues au pont redresseur à 6 diodes détériorent le courant statorique ainsi que le vecteur tension du réseau. Les paramètres du filtre doivent donc être choisis de manière à réduire le taux de distorsion harmonique de la tension de réseau à une valeur inférieure à $5 \%$. On peut tirer de (34) la fonction de transfert entre $\mathrm{I}_{\mathrm{g}}(\mathrm{s})$ et $\mathrm{V}_{\mathrm{g}}(\mathrm{s})$.

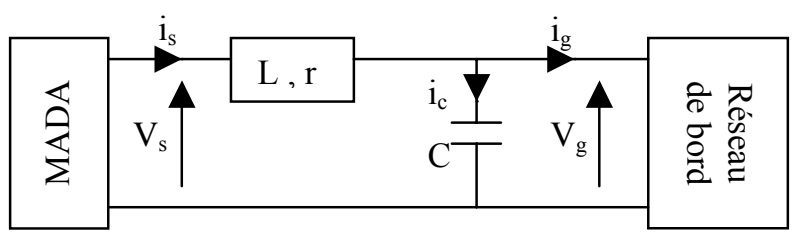

Fig. 12 Schéma équivalent monophasé du filtre LC

$$
\frac{V_{g}(s)}{I_{g}(s)}=\frac{(L s+r) \omega_{o}^{2}}{s^{2}+2 m \omega_{o} s+\omega_{o}^{2}}
$$

\section{B. Procédure de dimensionnement}

L'inductance du filtre est généralement dimensionnée égale à une fraction de l'impédance nominale du moteur, ainsi la chute de tension est réduite dans l'inductance du filtre. Dans ce cas, on choisira :

$$
\mathrm{L}=0.1 \mathrm{~L}_{\mathrm{s}}
$$

La résistance correspond, dans ce cas, à la résistance interne de l'inductance et est donc proportionnelle aux pertes Joules internes de l'inductance. Cette résistance crée donc des pertes au niveau du filtre. Dans ce cas, les pertes Joules sont définies inférieures à $1 \%$ de la puissance totale, donc le maximum acceptable de la résistance interne $r_{\max }$ peut être calculé de la manière suivante :

$$
\mathrm{r}_{\max }=\frac{0.01 \mathrm{P}_{\mathrm{s} \_ \text {nom }}}{3 \mathrm{I}_{\mathrm{s} \_ \text {nom }}^{2}}
$$

L'atténuation apportée par le filtre aux hautes fréquences est de $-20 \mathrm{~dB} /$ décade et elle dépend également du coefficient d'amortissement $\mathrm{m}$ imposé par l'utilisateur. La fréquence de coupure devrait être assez basse pour donner l'atténuation voulue et le coefficient d'amortissement assez grand pour augmenter cette atténuation. D'autre part, (34) montre qu'une fréquence de coupure trop basse pourrait résulter en de composants de grande taille [14]. De plus, un très grand coefficient d'amortissement entraînerait une valeur de résistance interne supérieure à $r_{\max }$. Il est donc nécessaire de trouver un compromis entre les dimensions du filtre et le TDH voulu.

D'après les composantes du courant harmonique décrites par (31), les caractéristiques amplitude-fréquence
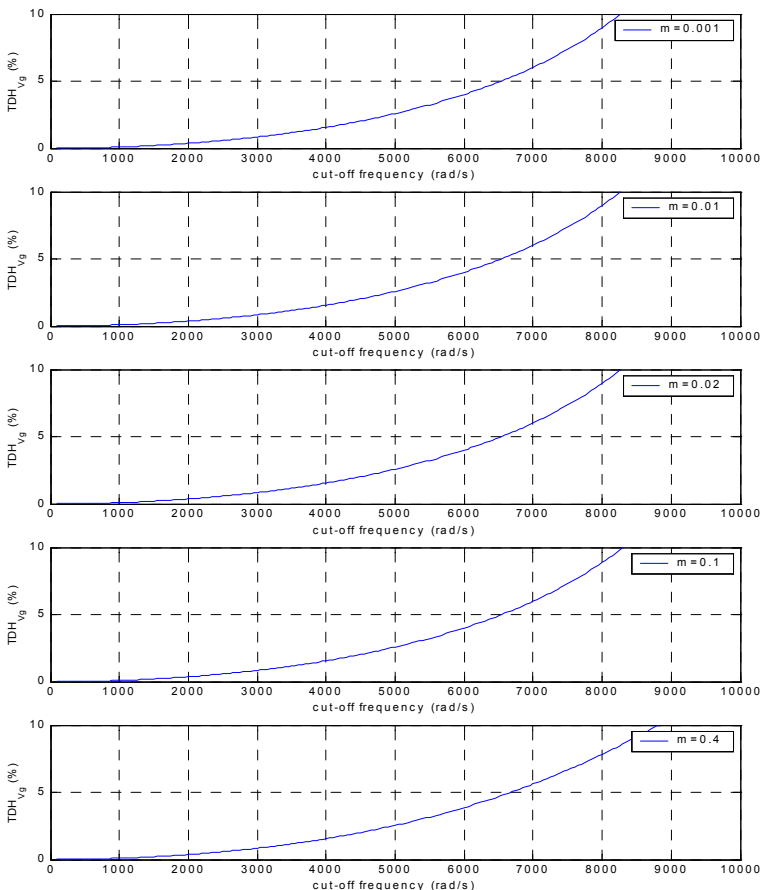

Fig. $13 \mathrm{TDH}$ de $\mathrm{V}_{\mathrm{g}}$ en fonction de la fréquence de coupure 

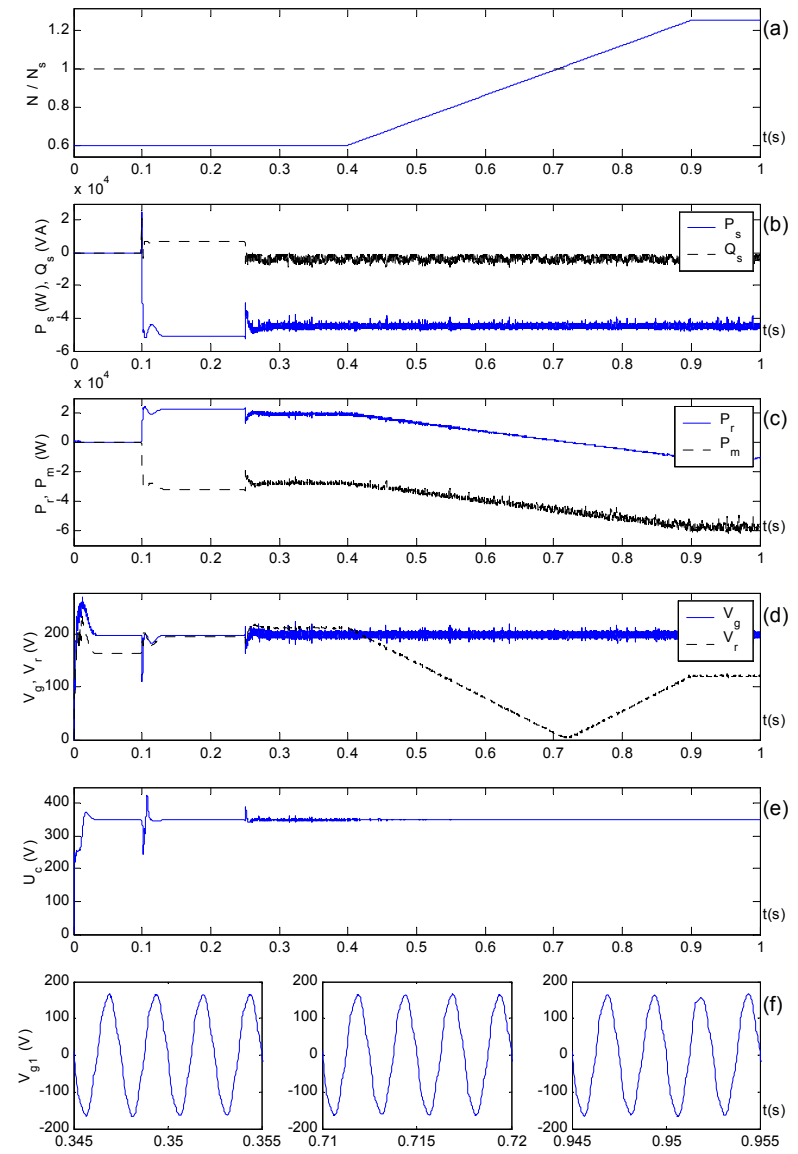

Fig. 14 Variation de charge NL jusqu'à 50\% suivie d'une variation de vitesse de $0.6 \mathrm{~N}_{\mathrm{s}}$ à $1.25 \mathrm{~N}_{\mathrm{s}}$ après filtrage, pour $50 \mathrm{kVA}$ de charge

données par (35) et sachant que l'amplitude de la tension du fondamental est de $115 \mathrm{~V}$, on peut calculer la relation entre la fréquence de coupure du filtre et le TDH de la tension de réseau après filtrage. Pour remplir les contraintes imposées par les équations (36) et (37), la figure 13 montre que le TDH reste inférieur à $5 \%$ pour une fréquence de coupure de $6300 \mathrm{rad} / \mathrm{s}$ avec un coefficient d'amortissement de 0.014 qui permet de ne pas dépasser $r_{\max }$.

Finalement, à partir de (35), connaissant $\omega_{0}$ et $\mathrm{L}$, on peut déduire la capacitance du filtre :

$$
C=\frac{1}{L \omega_{0}^{2}}
$$

\section{Résultats de simulation}

L'introduction d'un filtre LC avec une fréquence de coupure de $6300 \mathrm{rad} / \mathrm{s}$ pour réduire les harmoniques de courant est simulé de la même manière que précédemment. La figure 14 montre que la tension de réseau est presque sinusoïdale avec un TDH inférieur à $5 \%$ pour $50 \%$ de charges non-linéaires. Comparé au signal obtenu sans filtre (fig. 10), les oscillations sur les puissances active et réactive sont largement réduites par l'introduction du filtre et les critères du cahier de charge sont bien remplis.

\section{CONCLUSION ET TRAVAUX FUTURS}

Cet article présente un système VVFF pour des applications aéronautiques. Les principes de la commande vectorielle de la MADA et de la MSAP ont été présentés. Les résultats de simulation montrent que cette méthode permet d'obtenir une tension à fréquence et amplitude fixes sous une grande plage de variation de la vitesse d'entraînement de la turbine. L'ajout de charges nonlinéaires, comme les ponts redresseurs à diodes, introduisent des harmoniques qui détériorent les tensions du réseau. L'introduction d'un filtre LC du côté stator de la MADA permet de réduire ces harmoniques à un niveau acceptable selon le cahier de charges.

Dans un futur proche, l'étude du déséquilibre du système sera réalisée ainsi que l'optimisation du bus continu.

\section{REFERENCES}

[1] R. Pena, J.C. Clare, G.M. Asher, "Doubly-fed induction generator using back-to-back PWM converters and its application to variablespeed wind-energy generation", IEE Proc.-Electr.Power Appl., vol. 143, no. 3, pp. 231-241, May 1996.

[2] R. Pena, J.C. Clare, G.M. Asher, “A doubly-fed induction generator using back-to-back PWM converters supplying an isolated load from a variable-speed wind turbine", IEE Proc.-Electr.Power Appl., vol. 143, no. 5, pp. 380-387, Sept 1996.

[3] R. Pena, R. Cardenas, J.C. Clare, G.M. Asher, J. Rodriguez, P. Cortes, "Vector control of a diesel-driven doubly-fed induction machine for a stand-alone variable speed energy system", in Proc. IEEE IECON Conf., pp. 985-990, 2002.

[4] R. Pena, R. Cardenas, J.C. Clare, G.M. Asher, "Control strategy of a doubly-fed induction generators for a wind diesel energy system", in Proc. IEEE IECON Conf., pp. 3297-3302, 2002.

[5] M. Yamamoto, O. Motoyoshi, "Active and reactive power control for doubly-fed wound rotor induction generator", IEEE Trans. on Power Electron., vol. 6, no. 4, pp. 624-629, Oct 1991.

[6] R. Datta, V.T. Ranganathan, "A simple position-sensorless algorithm for rotor-side field-oriented control of wound-rotor induction generator", IEEE Trans. on Ind. Electron., vol. 48, no. 4, pp. 786-793, Aug 2001.

[7] Y. Tang, L. Xu, "A flexible active and reactive power control strategy for a variable speed constant frequency generating system", IEEE Trans. on Power Electron., vol. 10, no. 4, pp. 472-478, July 1995.

[8] S. Tnani, S. Diop, S.R. Jones, A. Berthon, "Novel control strategy of doubly-fed induction machines", Proc. Euro. Conf. on Power Electron. And App., pp. 1553-1558, Sevilla, Spain, 1995.

[9] S. Muller, M. Deicke, R.W. De Doncker, "Doubly-fed induction generator systems for wind turbines", IEEE Ind. App. Magazine, pp. 26-33, May/June 2002.

[10] T.H. Ortmeyer, W.U. Borger, "Control of cascaded doubly-fed machines for generator applications", IEEE Trans. on Power Appar. and Syst., vol. PAS-103, no. 9, pp. 2564-2571, Sept 1984.

[11] D. Forchetti, G. Guarcia, M.I. Valla, "Vector control strategy for a doubly-fed stand-alone induction generator", in Proc. IEEE IECON Conf., pp. 991-995, 2002.

[12] W. Leonhard, "Control of electrical drives", Springer, pp. 287-305, 1996.

[13] V. Oliveira, E. Monmasson, R. Meuret, J.P. Louis, "Steady state analysis of a doubly fed induction generator for aircraft application", in Proc. Electromotion Conf., pp. 547-551, June 2001.

[14] C. Xiyou, Y. Bin, G. Yu, "The engineering design and optimization of inverter output RLC filter in AC motor drive system", in Proc. IEEE IECON Conf., pp. 175-180, 2002.

[15] J.P. Besnard, G. Monroy, "Convertisseurs statiques de génération principale d'énergie dans l'aéronautique", in Proc. 3EI Conf., 1997 